\title{
Complications of mediastinal neural tumours
}

\author{
CHR IS T OP HER P A R IS H \\ Regional Thoracic Surgical Unit, Addenbrooke's and Papworth Hospitals, Cambridge ${ }^{1}$
}

Thirty-two mediastinal neural tumours were seen in the East Anglian Regional Thoracic Surgical Unit at Cambridge between October 1952 and July 1970. The descending order of frequency was neurofibroma, ganglioneuroma, neurilemmoma, neurofibrosarcoma, and neuroblastoma. The literature relating to these tumours is reviewed and the pathological and clinical complications encountered in this series and in the literature are described.

The East Anglian Regional Thoracic Surgical Unit at Papworth, near Cambridge, serves a population of approximately one-third of a million. From October 1952 to July 1970, 32 mediastinal neural tumours were diagnosed in the Unit. During the same period the Paediatric and Radiotherapeutic Units at Cambridge treated 18 (5 intra- and 13 extra-thoracic) neuroblastomas and two of the former have appeared in the 32 Papworth case series.

The material is divided into groups based on the following pathological definitions in order to study the frequency and type of complication.

Neurofibroma is a benign neoplasm arising from the non-specific nerve sheath. The neoplasm may be either solitary or multiple and has been known as von Recklinghausen's disease since 1882 but was originally described by Smith (1849). Neurilemmoma is a neoplasm derived from lemmocytes or specific nerve sheath cells and is otherwise known as Schwannoma or neurinoma. Ganglioneuroma or neurocytoma is derived from nerve cells. Pheochromocytoma is derived from chromaffin cells. The malignant variants of these four neoplasms are neurogenic sarcoma, neurilemmo sarcoma, neuroblastoma, and pheochromoblastoma. The non-chromaffin paraganglioma in the chest arising from aortic arch bodies is rare and none has been seen in the Papworth series.

The 32 surgical unit cases consisted of 18 neurofibromas, six ganglioneuromas, three neurilemmomas, three neurofibrosarcomas, one of which contained neurilemmomatous elements, and two neuroblastomas. There were no pheochromocytomas. The order of frequency is typical of that found in the literature emanating from thoracic

1Requests for reprints to : Christopher Parish, F.R.C.S., Thoraclc Surgical Unit, Papworth Hospital, Papworth Everard, Cambridge. surgical units. Our figures reflect the general con- $\bar{c}$ sensus on age distribution, namely 50 " of the cases of neuroblastoma occur in children under $\vec{\varphi}$ the age of 2 years, $50 \%$ of the ganglioneuromas are in children under 10 years, and $50 \%$ of the neurofibromas and neurilemmomas occur in patients in the third and fourth decades. In Bodian's (1963) collected series (from 1925 to 1962) $40 \%$ of 165 cases of neuroblastoma occurred $\stackrel{\mathcal{D}}{\mathcal{O}}$ in the first year of life with an equal male /female sex incidence. Morrison (1958) collected all the $\overrightarrow{\bar{O}}$ Liverpool region's mediastinal tumours and cysts and added to them the cases in the tumour register of the Society of Thoracic Surgeons. Of 332 such cases, 101 (that is, one-third) were neurogenic tumours. The location of the tumour is of importance in relation to its complications. $x$ Between $72 \%$ (Ringertz and Lidholm. 1956) and 3 92\% (Curreri and Gale, 1949) of neurogenic neoplasms occur in the posterior mediastinum. Our figures fall within this range and the reason may well be that the bulk of mediastinal neural tissue lies in the posterior mediastinum. Oosterwijk and Swierenga (1968), reporting 111 . cases of intrathoracic neurogenic tumours, stated $N_{\sigma}$ that these tumours have a predilection for the right side but elsewhere in the literature an equal frequency is quoted. In the Papworth series the $\mathrm{C}^{\omega}$ left side was slightly predominant but the Pap? worth series excluded non-mediastinal intra- $-\frac{0}{\square}$ thoracic neurogenic tumours.

The sex incidence in 794 cases collected by Cruickshank (1957) from the literature showed ato응 female/male ratio of $1 \cdot 79: 1$. In this series of $32 . \overrightarrow{\mathrm{D}}$ however, 17 were female and 15 male, a ratio which is reflected in the Oosterwijk and Swierenga $\varrho$ series of 59 women to 52 men.

The pathological complications were as follows: 8 acute inflammation and necrosis associated witho 
polymorphonuclear leucocyte infiltration ; chronic inflammation with lymphocytic infiltration; mucoid degeneration with histiocytic infiltration; as a result of acute inflammation and necrosis, chronic inflammation and mucoid degeneration; haemorrhage into the tumour occurred due to capillary failure; necrosis (especially in neuroblastoma); calcification with or without the preceding complications; cystic degeneration, the commonest form starting as a multicentric collection of microcysts and progressing to homogenous myxomucoid degeneration and even a total unilocular cyst ; and malignant degeneration.

Of the 32 Papworth cases five were malignant and 27 benign.

In Morrison's (1958) series $16(19 \%)$ were malignant and 85 benign, and in the Oosterwijk and Swierenga (1968) series $15(16 \%)$ were malignant and 96 benign. Blades (1946), however, quotes $3.3 \%$ and Efskind and Liavaag (1950) $20 \%$ as the percentage of malignant cases. The anterior mediastinal tumours are said to carry a high incidence of malignancy ; Morrison (1958) found one malignant out of three anterior mediastinal tumours, and Cruickshank (1957) collected 19 malignant tumours out of $57(33 \%)$ in nonposterior mediastinal sites in the chest; whereas $11.6 \%$ of 413 collected cases of intrathoracic neurogenic tumours were reported as malignant. Robb-Smith and Pennybacker (1952) state that the overall incidence of malignancy in von Recklinghausen's disease is difficult to assess and they think that the quoted figure of 10 to $15 \%$ is probably too high. In the Papworth series there were three cases of von Recklinghausen's neurofibromatosis, one of which was malignant. If we accept Cruickshank's figure of $11.6 \%$ malignancy in all primary intrathoracic neurogenic tumours we find that this parallels the rate to be found in neurofibromatosis at all sites. If, however, we add secondary tumours in the chest it becomes difficult or even impossible to produce any meaningful figure and indeed the published series shows a variation between 10 and $40 \%$ where malignant primaries and secondaries are included.

The clinical complications, for convenience, can be classified into pulmonary, neurological, alimentary, vascular, and miscellaneous.

Benign tumour presentation can be asymptomatic, as it was in 18 out of 32 cases in this series, all these being found by mass miniature radiographic examination or by routine preoperative chest radiological investigation prior to other forms of surgery. Horner's syndrome was found in two asymptomatic cases.
The pulmonary complications in the 14 symptomatic cases presenting in the benign series were dyspnoea, with either small or large tumours, pneumonia and empyema, tracheal compression and stridor, cough, and in one case distress on lying on the opposite side of the chest due to a $1050 \mathrm{~g}$ benign neurofibroma. One woman with a $1060 \mathrm{~g}$ tumour complained that she tilted to one side when swimming. Other symptoms recorded in the literature include haemoptysis in association with a benign anterior mediastinal neurilemmoma (Godwin et al., 1950) ; haemorrhagic effusion is quoted by Efskind and Liavaag (1950) as occurring with a benign tumour; and D'Abreu (1947) quoted recurrent pneumonia and 'asthmatic attacks' as well as emphysema, atelectasis, and tracheal obstruction with stridor. Hochberg, Griffin, and Bicunas (1950) described hypertrophic pulmonary osteoarthropathy which disappeared after excision of a neurofibrosarcoma of the diaphragm.

The neurological complications in the Papworth series consisted of pain due to pressure of the tumour on adjacent bone or intercostal nerves, Horner's syndrome, coldness, numbness, and pins and needles in the arm in plexiform tumours arising in the upper mediastinum and extending into the brachial plexus. Pain and loss of hot and cold sensation in the legs with sacral sparing occurred in a benign dumb-bell tumour. Pain was the presenting symptom in a pigmented neurilemmoma arising from the anterior aspect of the spinal cord and eroding two vertebral bodies. Recurrent nerve paralysis and epigastric pain and cramp are recorded (Gilbertsen and Lillehei, 1954) in tumours arising from the vagus nerve, these symptoms disappearing on removal of the benign tumour. The authors, however, state that there is no clear evidence that the growth promotes abnormal stimuli. Löblich (1952) referred to Pancoast's syndrome associated with an upper mediastinal benign tumour, and shoulder-tip pain is described by Weisel, Claudon, and Willson (1956) from a benign neurilemmoma lying on the diaphragm. Nanson (1957) described the associated meningocoele in von Recklinghausen's disease as accounting for $70 \%$ of all thoracic meningocoeles. These lead to additional pressure on the skeleton and pain with intercostal nerve compression and associated bone anomalies. Spinal cord complications due to the meningocoele are, however, uncommon.

The alimentary symptoms of neurogenic tumours in the Papworth series included dysphagia from a neurofibroma arising in the 
oesophagus and ulcerating into it. Another neurilemmoma in this series which caused tracheal compression also compressed the oesophagus and caused dysphagia. The epigastric symptoms associated with vagal nerve tumours have already been mentioned. Flatulence and vomiting have also occurred.

The vascular group of complications include compression of the superior vena cava in one of our cases, cardiac embarrassment, tachycardia, hypertension, and peripheral vasomotor phenomenon. Dizziness and hypotension are recorded in others.

Other complications include the presentation of a mass in the neck arising in the anterior mediastinum, or extension of a plexiform mediastinal tumour into the axilla. The presence of multiple subcutaneous nodules and skin pigmentation is related to the underlying disorder in von Recklinghausen's disease. Plenge (1928) described a $2 \frac{1}{2}$-year-old boy who presented with a lump in the neck which became sarcomatous at the age of 15 , leading to death; most of the tumour arose originally in the mediastinum. Pung and Hirsch (1955) described a plexiform mediastinal tumour in a 4-year-old girl which involved the heart and mediastinum and extended into the neck.

Rapid growth of the tumour radiologically with increasing symptomatology due to pressure was, in one of our cases, an indication of the onset of sarcomatous change; this patient, 10 years previously, had had a benign fibroma removed which was, however, reported as possibly showing evidence of early malignancy. She subsequently developed three further tumours on the right side weighing $991 \mathrm{~g}, 451 \mathrm{~g}$, and $6 \mathrm{~g}$. These consisted of neurofibroma, neurilemmoma, and frank fibrosarcoma in the largest tumour (Parish, 1957). Obvious symptoms of malignant spread to the glands in the neck and to subcutaneous sites occurred in one patient, and general symptoms of the onset of malignancy, for example anaemia and loss of weight, occurred in others.

In the neuroblastoma cases in children the pathological complication of necrosis in the tumour is common in the total Cambridge series and in the Bodian (1963) series. This leads to an influenza-like illness with fever, malaise, and vomiting followed by anaemia and is often diagnosed as infection. Diarrhoea is common. The neurological complications occur as in the adult, but hypertensive encephalopathy occurs in tumours containing large amounts of pressor amines. In Bodian's (1963) series of 58 cases in children 56 were dead one year after the onset of the illness. One spontaneous regression occurred, however, and this is of interest. The incidence of spontaneous remission is said to b? about $1 \%$ in the world literature (Rice, 1966 Bodian's (1963) case, a 4-month-old boy with mediastinal, liver, and skin deposits proven boj biopsy, was reported alive and well 15 years latef without treatment. Willis (1958) states that neuroblastic proliferation continues long after birth and probably as much as 10 years later. Permaneris clinical remission from 2 to 12 years occurre in 23 infants and in nine older children receiving vitamin $\mathbf{B}_{12}$ therapy in Bodian's series but not if any cases with bony secondary deposits. Simila treatment failed in all 31 older children. Masoin et al. (1957) reported the removal of a $50 \mathrm{~g}$ tumoum with $0.14 \mathrm{mg}$ of pressor amines per gramme of tumour from a 5-month-old infant, the tumou装 being a neuroblastoma.

Primary intrathoracic neuroblastomas have $\overrightarrow{\$}$ better prognosis than those arising in the abdomen (Claireaux and Williams, 1969), and there \& evidence (Koop, Kiesewetter, and Horn, 195 that the widest possible excision of the tumou even if incomplete, leads to an increased survivaf figure. Koop and Hernandez (1964) were unable to confirm Bodian's work on vitamin $B_{12}$. The two Papworth neuroblastoma cases are alive and wet several years after maximal excision and $B$ therapy. Other forms of treatment, chemotherapts and radiotherapy do not come within the scope of this paper.

Intrathoracic pheochromocytomas differ in no way from those arising elsewhere, according to the literature. There were no such cases in the Papworth series, in agreement with Cruickshank\& (1957) estimated figure of under $4 \%$ of all intra thoracic neurogenic tumours. Failure to recognize the nature of a tumour in the chest may lead to serious and fatal complications. Cone, Allen, an Pearson (1957) reported a child with severe hype tension leading to marked atheroma and intestinap haemorrhage with infarction secondary to occlit sive endarteritis. In adults the hypertension significant in most cases but its absence does not mean that a pheochromocytoma does not contai pressor substance (Maier and Humphreys, 1958\% At operation excessive manipulation of the tumour with liberation of pressor substances into the bloodstream leads to hypertensive crises. The marked vascular nature of the pleura over the tumour with bleeding on incision of the pleutid even at some distance from the tumour, the sof glandular, rather than hard nodular, state of the growth, and its dark brown colour should lead the 
surgeon to suspect its nature and to take steps to prevent these complications. Malignant secondaries from these tumours may also secrete pressor substances.

Finally, non-chromaffin paragangliomas arising from the aortic arch bodies are rare. This group probably contains tumours of a diverse range of pharmacological activity although having roughly similar histological appearances. Lattes (1950) states that they are not associated with hypertension but may even secrete a blood pressure lowering substance and have an antagonistic effect if they coexist with pheochromocytomas. The negative chromaffin reaction, however, may depend on the fixing and staining techniques, and some authors classify all non-adrenaline-secreting pheochromocytomas as paragangliomas. Maier and Humphreys (1958) state that each is similar from the histological and biological point of view. The pressor substances may well be found by chemical analysis of the tumour without hypertension having been present. Thus some paragangliomas in normotensives may well be pheochromocytomas.

Smithers and Gowing (1965) refer to tumours in the aortic arch region as chemodectomas. This term was suggested by Mulligan (1950) for a tumour of the chemoreceptors. Smithers and Gowing (1965) described two clinical types-first, those functioning as enlarged chemoreceptors leading to exaggeration of normal physiological responses with dizziness, blackouts, hypotension, and nystagmus after slight exertion or after minimal hyperventilation; and, secondly, some tumours containing cells producing noradrenaline. The mediastinal tumours of this nature are very rare. The 29th recorded chemodectoma in the aortic arch region occurred in a 61-year-old woman, compressing the trachea and causing choking attacks (Wilkinson and Forgan Smith, 1969).

I am indebted to Mr. B. B. Milstein for permission to include his cases.

\section{REFERENCES}

Blades, B. (1946). Mediastinal tumors; report of cases treated at Army Thoracic Surgery Centers in the United States. Ann. Surg., 123, 749.

Bodian, M. (1963). Neuroblastoma; an evaluation of its natural history and the effects of therapy, with particular reference to treatment with massive doses of Vitamin $\mathbf{B}_{12}$. Arch. Dis. Childh., 38, 606.

Claireaux, A. E., and Williams, D. I. (1969). In Recent Advances in Surgery, 7th ed., p. 62. ed. S. F. Taylor, London.

Cone, T. E., Allen, M. S., and Pearson, H. A. (1957). Pheochromocytoma in children. Pediatrics, 19, 44.

Cruickshank, D. B. (1957). Primary intrathoracic neurogenic tumours. J. Fac. Radiol. (Lond.), 8, 369.
Curreri, A. R., and Gale, J. W. (1949). Mediastinal tumors. Arch. Surg., 58, 797.

D'Abreu, A. L. (1947). Thoracic neurofibroma. Brit. J. Tuberc., 41, 55.

Efskind, L., and Liavaag, K. (1950). Intrathoracic neurogenic tumors. J. thorac. Surg., 20, 13.

Gilbertsen, V. A., and Lillehei, C. W. (1954). Bilateral intrathoracic neurofibromas of the vagus nerves. J. thorac. Surg., 28, 78.

Godwin, J. T., Watson, W. L., Pool, J. L., Cahan, W. G., and Nardiello, V. A. jun. (1950). Primary intrathoracic neurogenic tumors. J. thorac. Surg., 20, 169.

Hochberg, L. A., Griffin, E. H., and Bicunas, A. (1950). Neurofibrosarcoma of the anterior mediastinum. Surgical removal. J. thorac. Surg., 20, 315.

Koop, C. E., and Hernandez, J. R. (1964). Neuroblastoma: experience with 100 cases in children. Surgery, 56, 726.

- Kiesewetter, W. B., and Horn, R. C. (1955). Neuroblastoma in childhood. Surgery, 38, 272.

Lattes, R. (1950). Nonchromaffin paraganglioma of ganglion nodosum, carotid body, and aortic-arch bodies. Cancer (Philad.), 3, 667.

Löblich, H. J. (1952). Die neurogene Gruppe der Tumoren mit Pancoast-Syndrom. Z. Krebsforsch., 58, 576.

Maier, H. C., and Humphreys, G. H. (1958). Intrathoracic pheochromocytoma. J. thorac. Surg., 36, 625.

Mason, G. A., Hart-Mercer, J., Millar, E. J., Strang, L. B., and Wynne, N. A. (1957). Adrenaline-secreting neuroblastoma in an infant. Lancet, 2, 322.

Morrison, I. M. (1958). Tumours and cysts of the mediastinum. Thorax, 13, 294.

Mulligan, R. M. (1950). Chemodectoma in the dog. Amer. J. Path., 26, 680.

Nanson, E. M. (1957). Thoracic meningocele associated with neurofibromatosis. J. thorac. Surg., 33, 650.

Oosterwijk, W. M., and Swierenga, J. (1968). Neurogenic tumours with an intrathoracic localization. Thorax, 23, 374.

Parish, C. (1957). The clinical features of intrathoracic neurogenic tumours. J. Fac. Radiol. (Lond.), 8, 381.

Plenge, K. (1928). Über eine eigenartige plexiforme Neubilding des Nervensystems in der Gegend des Halses und der Halswirbelsäule. Virchows Arch. path. Anat., 269, 83.

Pung, S., and Hirsch, E. F. (1955). Plexiform neurofibromatosis of the heart and neck. Arch. Path., 59, 341.

Rice, M. S. (1966). Neuroblastoma in childhood. Aust. paediat. J., 2, 1.

Ringertz, N., and Lidholm, S. O. (1956). Mediastinal tumors and cysts. J. thorac. Surg., 31, 458.

Robb-Smith, A. H. T., and Pennybacker, J. (1952). The British Encyclopaedia of Medical Practice, vol. 10, 2nd ed., p. 549.

Smith, R. W. (1849). A Treatise on the Pathology, Diagnosis, and Treatment of Neuroma. Hodges and Smith, Dublin.

Smithers, D. W., and Gowing, N. F. C. (1965). Chemodectoma in the region of the aortic arch. Thorax, 20, 182.

Weisel, W., Claudon, D. B., and Willson, D. M. (1956). Neurilemmoma of the diaphragm. J. thorac. Surg., 31, 750.

Wilkinson, R., and Forgan-Smith, R. (1969). Chemodectoma in relation to the aortic arch (aortic body tumour); a clinical report. Thorax, 24, 488.

Willis, R. A. (1958). The Borderland of Embryology and Pathology, p. 292. Butterworth, London. 